\title{
QI hub - creating a culture of quality improvement
}

\author{
Authors: Alex Bunn, ${ }^{\mathrm{A}}$ Mridula Rajwani, ${ }^{\mathrm{A}}$ Sahana $\mathrm{Rao}^{\mathrm{A}}$ and Joanne Bunyan ${ }^{\mathrm{A}}$
}

\section{Introduction}

Staff work tirelessly to deliver high quality care in demanding settings, and are keen to further improve services, but often are short of time, support or skills to do so. ${ }^{1}$ Experience and feedback has shown that formal support for developing quality improvement (QI) skills is often fragmented and difficult to access. ${ }^{2}$

At our organisation there are excellent pockets of activity, but limited access to opportunities for shared learning. Our aim was to bring together this activity, while developing a community of empowered individuals and teams that drives a culture of continuous, sustained improvement within their divisions for the benefit of patients, colleagues and departments.

\section{Methods}

The QI Hub delivers a central programme open to all within the trust who are keen to undertake improvement. We support them through their QI journey by equipping them with skills, tools and a structure to lead projects. ${ }^{3}$

The format consists of an educational programme running in 3-monthly cycles providing fortnightly, dedicated QI education delivered by an experienced faculty. Participants take part in individualised, practical workshops together with face-toface sessions and peer support. Model for improvement, Lean methodology and process mapping are just a few of the topics covered. This culminates in a presentation event which provides an opportunity to share project ideas and progress with the team, faculty and trust leadership.

Two cohorts have been run thus far.

\section{Results and discussion}

The programme was highly popular, with representation from several divisions and across a wide-ranging selection of multidisciplinary healthcare professionals (Table 1; Fig 1).

Feedback was collected using a Likert Survey before and after the programme, which demonstrated consistently positive outcomes. These are demonstrated in Fig 2.

The majority of participants had not had teaching or previously been involved in QI prior to the programme. All but one felt they had support from line managers and divisional leaders when carrying out their project. Encouragingly, $100 \%$ of responding

\footnotetext{
Authors: ${ }^{A}$ Oxford University Hospitals NHS Trust, Oxford, UK
}

\begin{tabular}{ll}
$\begin{array}{l}\text { Table } 1 . \text { Multidisciplinary healthcare professionals } \\
\text { taking part in the programme }\end{array}$ & Number \\
\hline Staff & 4 \\
Consultants & 16 \\
Junior doctors & 2 \\
Physician associates & 8 \\
Therapists & 4 \\
Pharmacists & 5 \\
Nurses & 1 \\
Admin staff & 1 \\
Governance staff & 2 \\
Dieticians &
\end{tabular}

delegates would recommend the programme to colleagues, with more than $90 \%$ enjoying attending the sessions delivered.

After the completion of the second cohort, we will repeat the collection of feedback in order to strengthen the validity of current analysis, and also plan to complete an impact assessment in the near future.

\section{Conclusion}

We have successfully achieved our aims and demonstrated increased understanding and engagement with quality

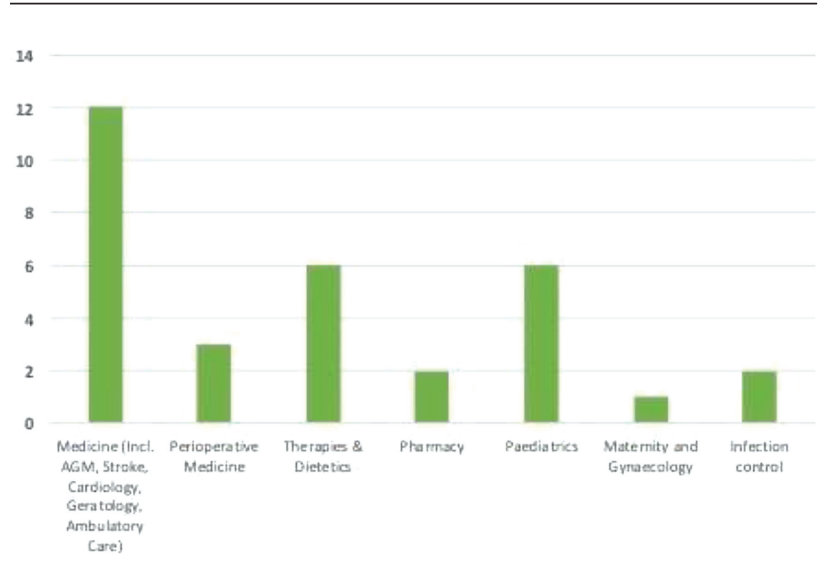

Fig 1. Graph displaying the number of programme delegates representing different departments. 


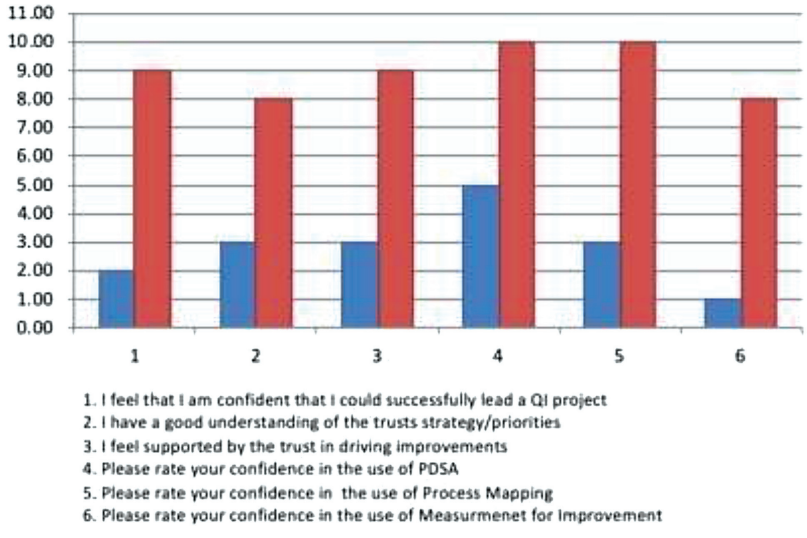

Fig 2. Survey responses before and after attending the programme. Bars show number of respondents agreeing (responses 1-3 on a Likert scale from 1 denoting strongly agree to 7 denoting strongly disagree) with the statement before (blue) and after (red) the programme.

improvement. These outcomes are in keeping with the trust's key priority to improve our safety culture, and we look forward to developing the programme further.
We are currently in the second cohort of the programme with an increased number of delegates, as well as wider engagement from the trust senior management team.

Future aims include creating a platform for shared learning, partnering with Oxford University and the Deanery to incorporate QI education into the curriculum, and expanding our programme to connect with colleagues within the primary care sector. We hope to develop an online platform for alumni to stay connected, and share examples of exemplar activity that could be replicated elsewhere.

\section{Conflicts of interest}

None declared.

\section{References}

1 Jones B, Woodhead T. Building the foundations for improvement: how five UK trusts built quality improvement capability at scale within their organisations. The Health Foundation, 2015.

2 Worsley C, Webb S, Vaux E. Training healthcare professionals in quality improvement. FHJ 2016:3:207-10.

3 The Health Foundation. Quality Improvement training for healthcare professionals. The Health Foundation, 2012. www.health. org.uk/publications/quality-improvement-training-for-healthcareprofessionals [Accessed 30 October 2019]. 